Supporting Information for:

\title{
SERS Study of the Mechanism of Plasmon-Driven Hot Electron Transfer Between Gold Nanoparticles and PCBM
}

Yue Wu, Muwen Yang, Tyler W. Ueltschi, Martín A. Mosquera, Zhu Chen, George C.

Schatz*, and Richard P. Van Duyne

Department of Chemistry, Northwestern University, Evanston, Illinois 60208, United States

*Email: g-schatz@northwestern.edu 


\section{The Extinction spectra of gold nanoparticles}

Figure S1 shows the UV-vis extinction spectra of gold nanoparticles before and after the sample preparation. The monomer plasmon resonance (around $539 \mathrm{~nm}$ ) did not change before and after the aggregation (Figure S1), showing that the gold nanoparticles were not over-aggregated.

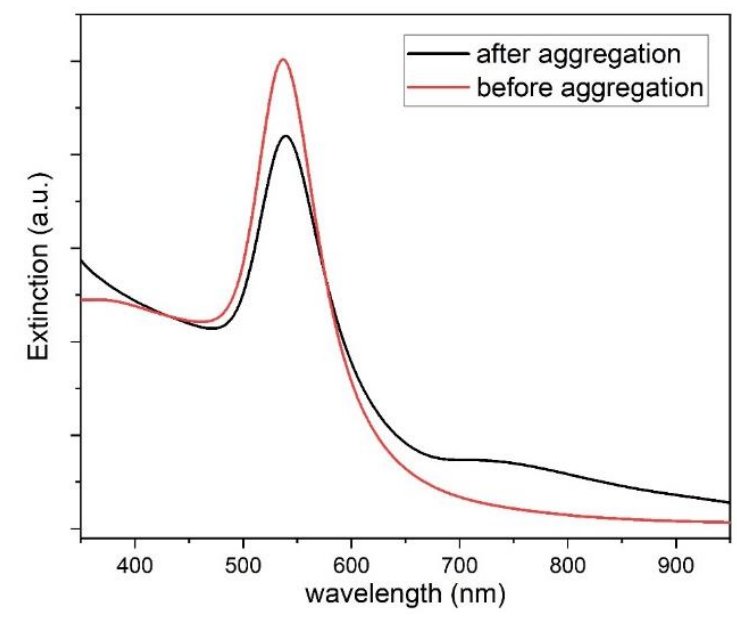

Figure S1. UV-vis extinction spectra of gold nanoparticles before and after aggregation.

\section{The CW pump-probe SERS setup}

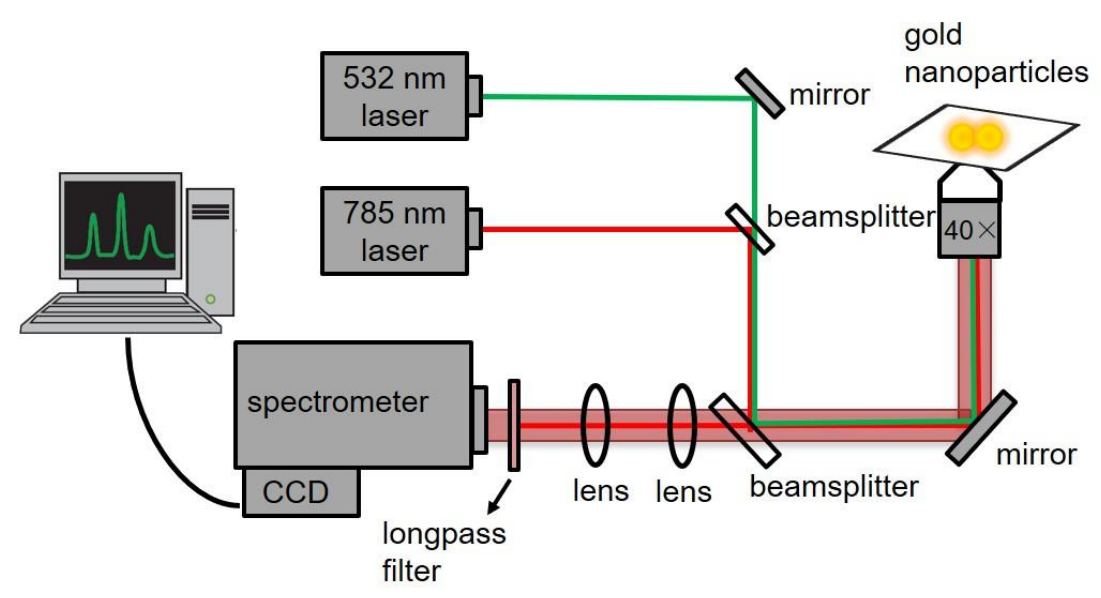

Figure S2. CW pump-probe SERS setup. 


\section{Control experiments and detailed data analysis}

1. Control experiments to confirm $785 \mathrm{~nm}$ probe laser is a good SERS probe for this system.

Several control experiments were performed to confirm that the peak shift of PCBM pentagonal pinch mode is not affected by the $785 \mathrm{~nm}$ probe laser. Figure S3a shows a time trace of PCBM SER spectra under $785 \mathrm{~nm}$ probe laser illumination. There was no peak shift with $785 \mathrm{~nm}$ probe laser only on the gold nanoparticle/PCBM samples. Figure S3b shows PCBM SER spectra under various probe power. The peak position and linewidth didn't change with the increase of probe power. These control experiments demonstrate that $785 \mathrm{~nm}$ laser is a good SERS probe for the gold nanoparticle aggregates with PCBM molecules.
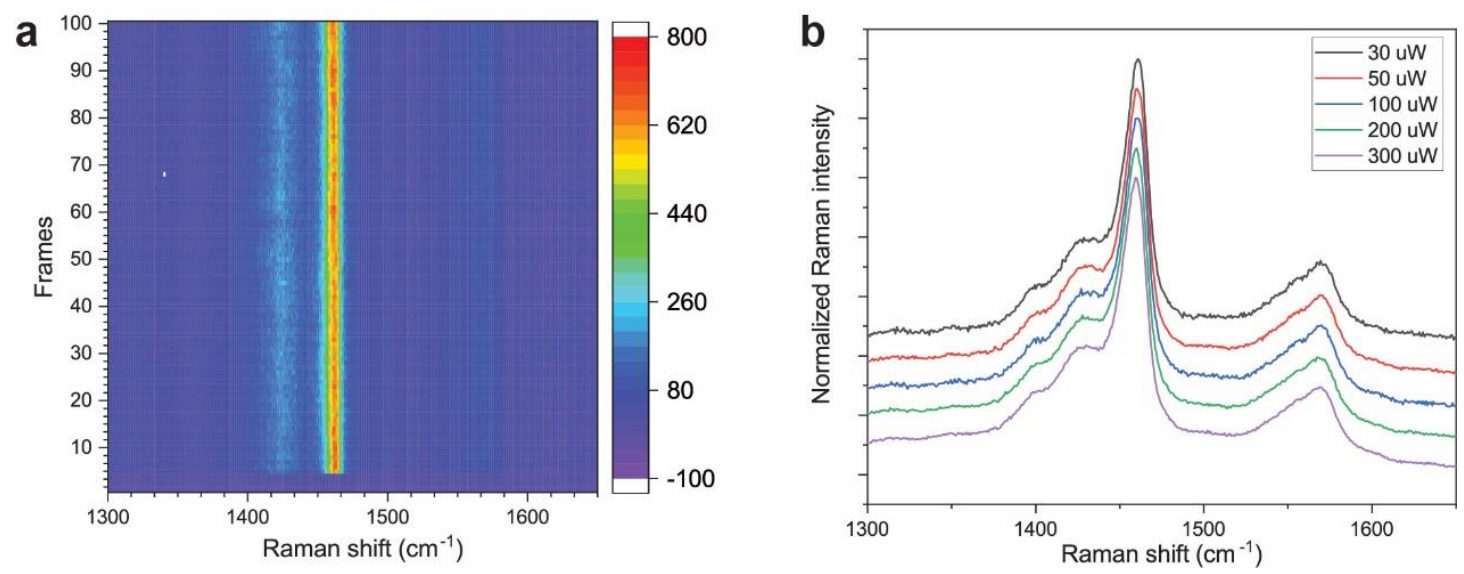

Figure S3. (a) continuous collection of PCBM SER spectra under the $785 \mathrm{~nm}$ probe laser only. The probe power was $100 \mu \mathrm{W}$. (b) The PCBM SER spectra under the 785 nm probe laser with different laser power. 
2. Degradation of PCBM under high pump laser power.

When the pump laser power is too high, PCBM molecules in the gold nanoparticles "hot spots" can go through photodegradation. The SER spectrum of photodamaged PCBM has the feature of significantly decreased Raman signal, large peak broadening and the rising of new peaks. Figure S4 shows a typical PCBM SER spectrum when pump laser is too high.

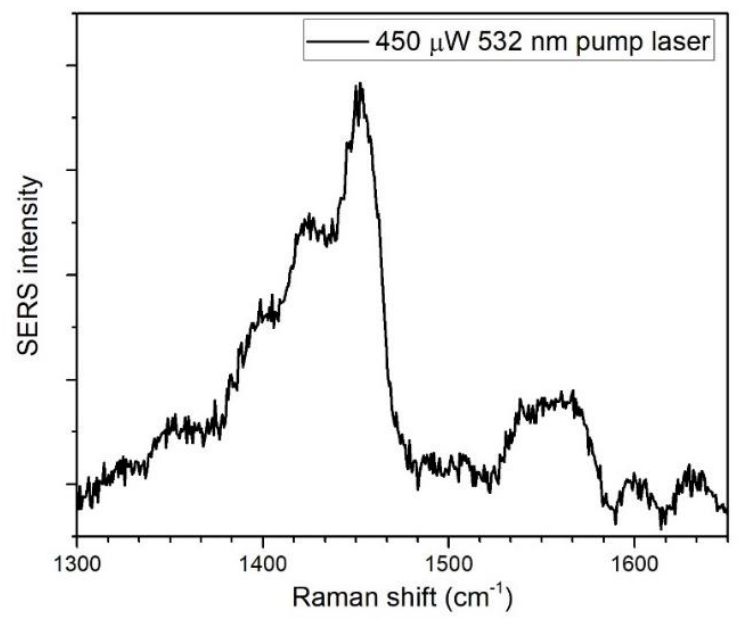

Figure S4. Photodegraded PCBM SER spectrum under $450 \mu \mathrm{W} 532 \mathrm{~nm}$ pump laser.

\section{Peak broadening.}

Besides the peak shift of the PCBM pentagonal pinch mode under pump laser illumination, a slight peak broadening behavior was also observed. Figure S5 shows the trend of the full width at half maximum (FWHM) of the pentagonal pinch mode under different pump laser power. The peak broadening is not very significant and the FWHM of the broadest peak is around $25 \mathrm{~cm}^{-1}$, which is within the normal range of a Raman peak. More interestingly, Figure S3b shows that the FWHM of the Raman peaks remained unchanged under $785 \mathrm{~nm}$ probe laser only. It indicates that the slight 
peak broadening is also an effect of the pump laser. One possible explanation is that with the increasing pump laser power, the gold nanoparticles bear more charge which induces the inhomogeneous broadening of the Raman peak. This can also be considered as an indirect evidence of charge transfer between gold nanoparticles and PCBM molecules.

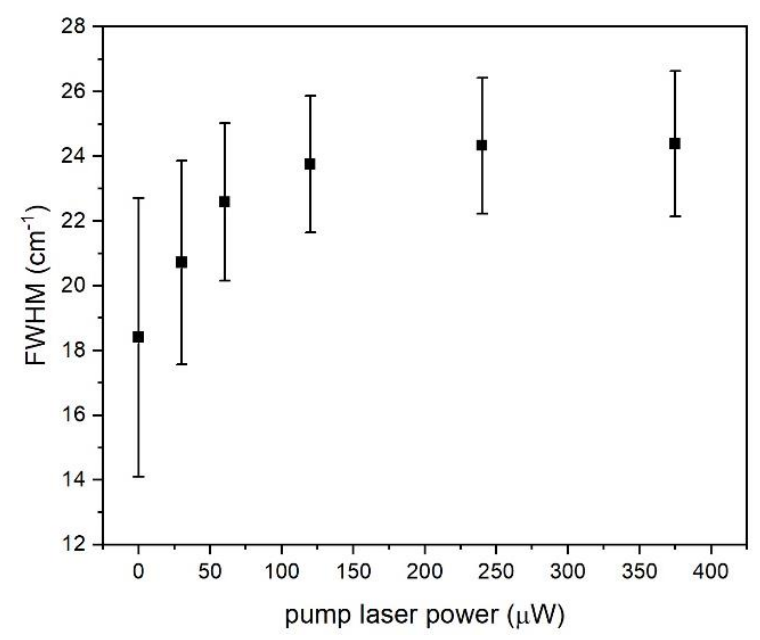

Figure S5. The FWHM of PCBM pentagonal pinch Raman mode with increasing pump laser power.

\section{Two-Gaussians fitting.}

Here we compare one-Gaussian fitting (used in main text) and two-Gaussian fitting for PCBM SER spectra under the illumination of pump laser. The idea of two-Gaussian fitting is to fit the Raman modes of two species: PCBM neutral species and PCBM anions. The conclusion is that two-Gaussian does not provide more insightful information than one-Gaussian fitting and sometimes leads to confusing results. Here are the details: 
1) The two-Gaussian fitting with two fixed Raman peak positions of PCBM anions derived from theoretical calculations failed to give reasonable results. DFT calculations predict that the frequency shift $(\Delta v)$ of the PCBM pentagonal pinch mode is around $6.5 \mathrm{~cm}^{-1}$. However, if we fix the relative difference of the peak positions of two Gaussians at $6.5 \mathrm{~cm}^{-1}$, the two-Gaussians fitting for some of the PCBM SER spectra, especially at higher pump power, fail to converge (Figure S6). If we relax the condition of fixed $\Delta v$, the $\Delta v$ obtained from two-Gaussian fitting in Figure S6 is 8.7 $\mathrm{cm}^{-1}$. By performing the two-Gaussians fitting for different PCBM SER spectra, we found that $\Delta v$ varies with pump laser power and different samples, which did not provide more information than a one-Gaussian fitting. For the same reasons, we were not able to calculate the "percent reduction" by doing two-Gaussian fitting with calculated frequencies of PCBM neutral species and anions.

2) The two-Gaussian fitting cannot explain the Raman peak width broadening. Figure R2 is the comparison between one-Gaussian fitting and two-Gaussians fitting for the same PCBM SER spectrum under $150 \mu \mathrm{W}$ pump laser power. For one-Gaussian fitting, the peak position is $1453.4 \mathrm{~cm}^{-1}$ and FWHM is $16.0 \mathrm{~cm}^{-1}$. It seems like FWHM is slightly broadened compared to the unpumped SER spectrum $\left(11.2 \mathrm{~cm}^{-1}\right)$. However, the FWHM of the two-Gaussian fitting for the anion part is $16.2 \mathrm{~cm}^{-1}$, which is similar to one-Gaussian fitting result. 


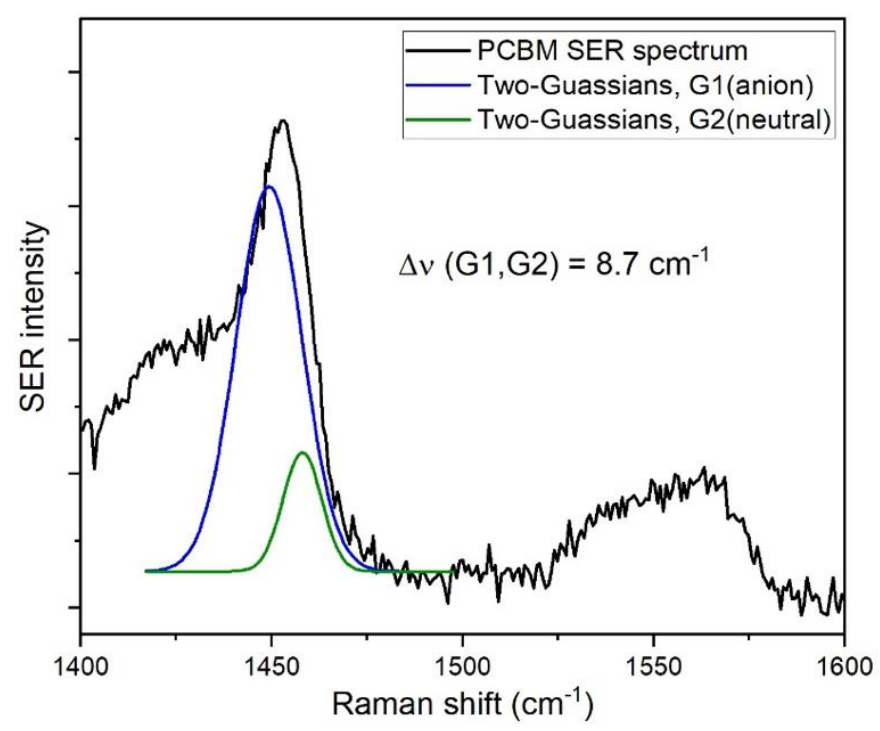

Figure S6. PCBM SER spectrum under $375 \mu \mathrm{W} 532 \mathrm{~nm}$ pump laser (black curve).

The blue and green curves are two parts of the two-Gaussian fitting.

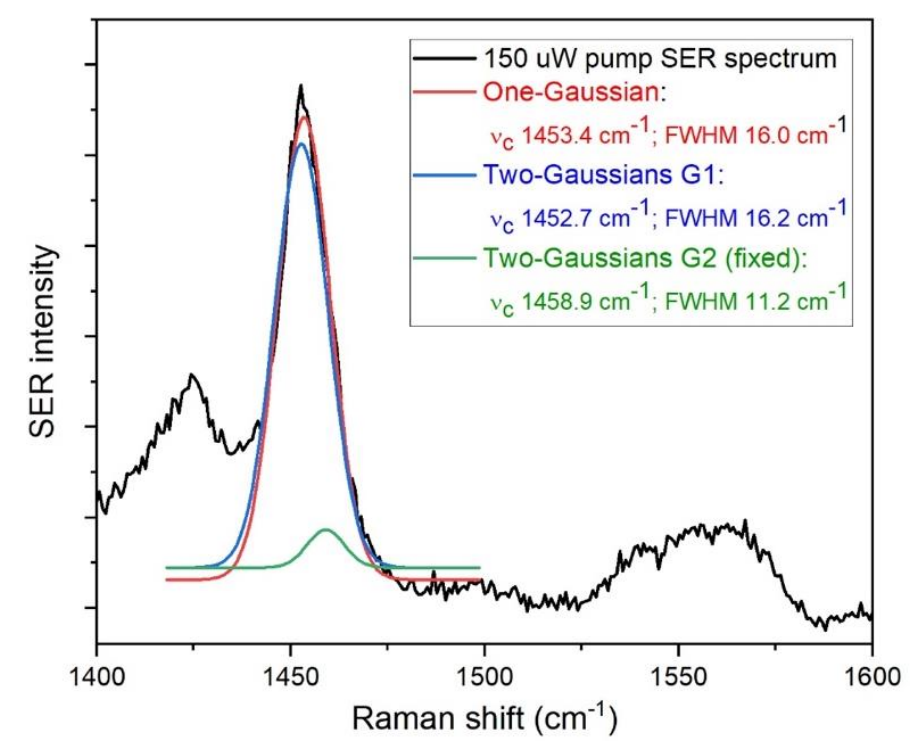

Figure S7. SER spectrum of PCBM under a $532 \mathrm{~nm}$ pump laser with $150 \mu \mathrm{W}$ power (black curve) and the comparison between one-Gaussian (red curve) and two-Gaussians fitting (blue curve and green curve). 
5. Comparison of PCBM SER spectra with pump laser on and off.

Here the three frames before (Frame \#20), during (Frame \#40) and after (Frame \#80) from the waterfall plot of Figure 1b is plotted for comparison, shown as Figure S8. The signal-to-noise ratio is not as good as Figure 1c because the acquisition time for each frame is only 2 seconds. In Figure S8, we can clearly see the peak shift when the pump laser was turned on. There was no significant Raman intensity decrease during the experiment, indicating little or no photodamage to PCBM molecules. In this plot after the pump was turned off, the pentagonal pinch mode reversibly shifted back to the original frequency.

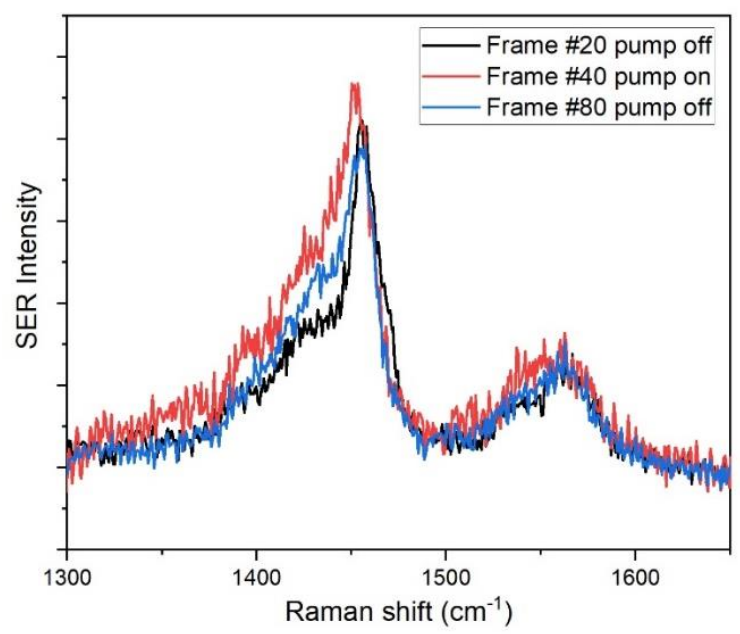

Figure S8. The SER spectra of PCBM extracted from the waterfall plot of Figure $1 \mathrm{~b}$ (Frame \#20, \#40 and \#80).

The derivation of the kinetic model for pump power dependence experiment. 
Considering the elementary electron transfer reaction:

$$
\mathrm{PCBM}+\mathrm{e}^{-} \underset{k_{-1}}{\stackrel{k_{1}}{\rightleftharpoons}} \mathrm{PCBM}^{-}
$$

The concentration change rate of $\mathrm{PCBM}^{-}$can be expressed as follows:

$$
\frac{\mathrm{d}\left[\mathrm{PCBM}^{-}\right]}{\mathrm{d} t}=k_{1}[\mathrm{PCBM}]\left[\mathrm{e}^{-}\right]-k_{-1}\left[\mathrm{PCBM}^{-}\right]
$$

Here we assume the total PCBM concentration in gold nanoparticle hot spots is constant: $[\mathrm{PCBM}]+\left[\mathrm{PCBM}^{-}\right]=[\mathrm{PCBM}]_{0}$. The concentration of photogenerated electrons $\mathrm{e}^{-}$is proportional to the photon flux, namely the pump laser intensity $I_{\mathrm{L}}$ :

$$
\left[\mathrm{e}^{-}\right] \propto \mathrm{n} \square v \propto I_{\mathrm{L}}
$$

$\mathrm{n}$ is the number of photons. Based on the steady state approximation $\frac{\mathrm{d}\left[\mathrm{PCBM}^{-}\right]}{\mathrm{d} t}=0$, eq 1 can be rewritten as follows with the ratio $\theta$ :

$$
\theta=\frac{\left[\mathrm{PCBM}^{-}\right]}{\left[\mathrm{PCBM}_{0}\right.}=\frac{\frac{k_{1}}{k_{-1}}\left[\mathrm{e}^{-}\right]}{1+\frac{k_{1}}{k_{-1}}\left[\mathrm{e}^{-}\right]}=\frac{K_{\mathrm{eq}}^{\prime} I_{\mathrm{L}}}{1+K_{\mathrm{eq}}^{\prime} I_{\mathrm{L}}}
$$

\section{PCBM full cyclic voltammetry (CV)}

Figure $\mathrm{S} 9$ shows the full $\mathrm{CV}$ of $\mathrm{PCBM}$ in the range of $-2.4 \mathrm{~V}$ to $1.4 \mathrm{~V} v s$. $\mathrm{Fc} / \mathrm{Fc}^{+}$. At the positive potential side there is an irreversible oxidation peak, indicating that the PCBM cation is not very stable. 


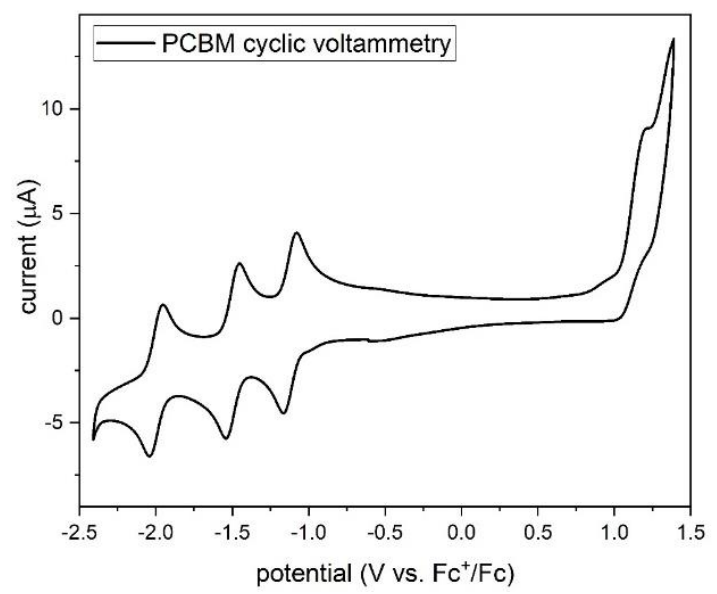

Figure S9. Full cyclic voltammetry of $2 \mathrm{mmol} / \mathrm{L} \quad \mathrm{PCBM}$ in $o$-dichlorobenzene/acetonitrile (5:1 by volume) with $0.1 \mathrm{~mol} / \mathrm{L} \mathrm{TBAPF}_{6}$ at $100 \mathrm{mV} / \mathrm{s}$.

\section{Additional Information of DFT simulations of the PCBM-Au system.}

Electronic structure calculations for $\mathrm{PCBM}$ interacting with a $\mathrm{Au}_{20}$ cluster were performed using the Amsterdam Density Functional (ADF) program. ${ }^{1}$ The basis set TZP and exchange-correlation functional BP86 were used. The zero-order regular approximation was used to include the relativistic effect for the calculations. Lowest 30 allowed excitation transitions were calculated. The lowest singlet transition at 1.04 $\mathrm{eV}$, HOMO to LUMO, is plotted in Figure S10, showing clear charge-transfer character (from gold to PCBM).

Vibrational mode calculations for fullerene and different ion species were performed using the NWChem program. ${ }^{2}$ Two different methods were used: the B3LYP functional with the 6-311G* basis set and the BP86 functional with the TZVP basis set. Both methods give similar results. Table S1 lists all the calculated vibrational frequencies of the four $\mathrm{C}_{60}$ species using B3LYP functional. In the case of 
BP86 functional with TZVP basis set, the Raman differential cross section $d \sigma / d \Omega$ of $\mathrm{C}_{60}$ was also calculated (Figure $\mathrm{S} 11$ ). The Raman response of open-shell $\mathrm{C}_{60}{ }^{-}$cannot be directly calculated with NWChem, so its Raman differential cross section is approximated with the Raman differential cross section of the corresponding mode of neutral $\mathrm{C}_{60}$. The stick spectra are broadened using Lorentzian lineshape with FWHM of $20 \mathrm{~cm}^{-1}$.

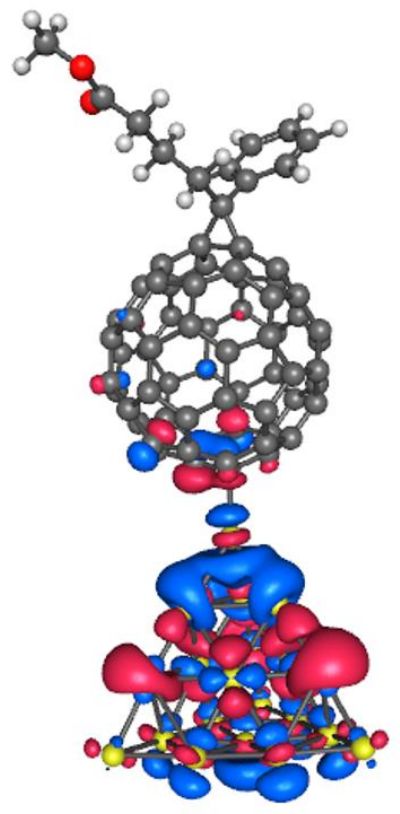

HOMO

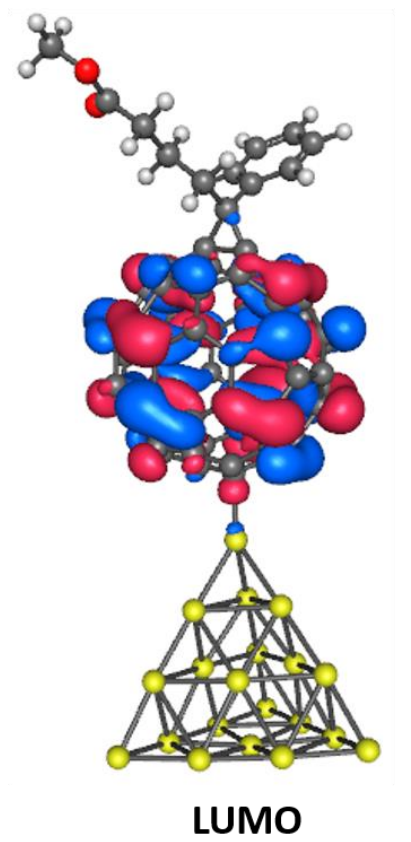

Figure S10. DFT calculation of PCBM and $\mathrm{Au}_{20}$ cluster. The HOMO-LUMO transition shows clear charge-transfer character. 


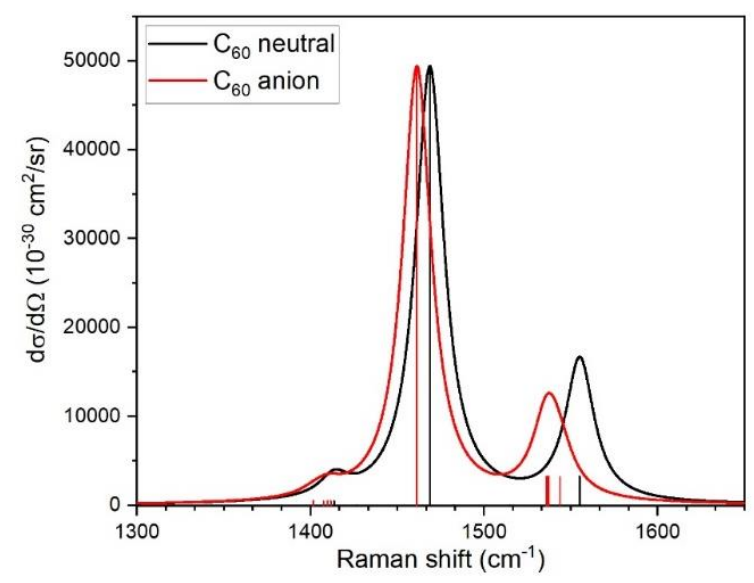

Figure S11. The Raman differential cross section of $\mathrm{C}_{60}$ (black) and $\mathrm{C}_{60}{ }^{-}$(red) calculated using DFT method. The Raman modes were broadened with Lorentzian lineshape $\left(\mathrm{FWHM}=20 \mathrm{~cm}^{-1}\right)$ to simulate the Raman spectra.

Table S1. DFT Vibrational Frequency Calculations of Different $\mathrm{C}_{60}$ Species with B3LYP

Vibrational frequencies of different $\mathrm{C}_{60}$ species $^{a}\left(\mathrm{~cm}^{-1}\right)$

\begin{tabular}{ccccc}
\hline modes & $\mathbf{C}_{\mathbf{6 0}}$ & $\mathbf{C}_{\mathbf{6 0}}{ }^{+}$ & $\mathbf{C}_{\mathbf{6 0}}{ }^{-}$ & $\mathbf{C}_{\mathbf{6 0}}{ }^{2-}$ \\
$\mathbf{1}$ & 264.7 & 209.7 & 255.8 & 237.7 \\
$\mathbf{2}$ & 265.4 & 222.5 & 256.4 & 241.6 \\
$\mathbf{3}$ & 266.8 & 251.9 & 261.2 & 264.9 \\
$\mathbf{4}$ & 267.1 & 264.9 & 262.9 & 265.2 \\
$\mathbf{5}$ & 267.5 & 265.7 & 263.7 & 265.9 \\
$\mathbf{6}$ & 344.6 & 342.8 & 339.1 & 335.5 \\
$\mathbf{7}$ & 345.0 & 343.9 & 343.1 & 347.9 \\
$\mathbf{8}$ & 345.7 & 347.3 & 343.7 & 349.7
\end{tabular}




\begin{tabular}{|c|c|c|c|c|}
\hline 9 & 358.0 & 353.4 & 353.9 & 350.0 \\
\hline 10 & 358.3 & 353.8 & 354.9 & 350.6 \\
\hline 11 & 359.0 & 355.9 & 355.3 & 356.7 \\
\hline 12 & 359.6 & 358.5 & 355.5 & 360.0 \\
\hline 13 & 408.5 & 400.3 & 397.5 & 378.6 \\
\hline 14 & 409.1 & 404.8 & 402.6 & 388.8 \\
\hline 15 & 409.2 & 405.3 & 402.6 & 395.5 \\
\hline 16 & 409.6 & 406.7 & 405.8 & 396.5 \\
\hline 17 & 409.7 & 407.4 & 407.0 & 399.1 \\
\hline 18 & 439.9 & 423.8 & 408.2 & 406.1 \\
\hline 19 & 440.6 & 431.3 & 409.7 & 410.7 \\
\hline 20 & 442.1 & 434.0 & 433.9 & 426.5 \\
\hline 21 & 442.6 & 436.7 & 440.5 & 438.9 \\
\hline 22 & 443.1 & 442.5 & 441.5 & 446.4 \\
\hline 23 & 490.0 & 480.3 & 485.7 & 483.2 \\
\hline 24 & 490.9 & 487.3 & 487.1 & 487.6 \\
\hline 25 & 492.8 & 495.3 & 487.8 & 489.5 \\
\hline 26 & 493.0 & 495.5 & 487.9 & 490.3 \\
\hline 27 & 496.9 & 497.2 & 496.5 & 495.6 \\
\hline 28 & 543.0 & 538.5 & 517.9 & 495.6 \\
\hline 29 & 544.1 & 539.1 & 526.6 & 518.5 \\
\hline 30 & 544.8 & 541.3 & 529.5 & 526.0 \\
\hline 31 & 545.4 & 542.7 & 530.9 & 527.8 \\
\hline 32 & 545.6 & 545.0 & 536.5 & 531.3 \\
\hline 33 & 545.9 & 545.8 & 536.9 & 533.5 \\
\hline 34 & 546.0 & 549.9 & 538.6 & 535.5 \\
\hline 35 & 546.7 & 555.8 & 541.6 & 539.5 \\
\hline 36 & 567.4 & 565.9 & 563.3 & 553.6 \\
\hline 37 & 568.4 & 566.8 & 564.7 & 555.0 \\
\hline
\end{tabular}




\begin{tabular}{|c|c|c|c|c|}
\hline 38 & 568.9 & 567.9 & 564.8 & 562.7 \\
\hline 39 & 578.1 & 578.1 & 566.5 & 564.4 \\
\hline 40 & 578.4 & 578.5 & 568.6 & 565.6 \\
\hline 41 & 578.6 & 578.9 & 573.5 & 575.9 \\
\hline 42 & 578.7 & 580.1 & 576.0 & 578.5 \\
\hline 43 & 580.2 & 580.7 & 578.9 & 580.1 \\
\hline 44 & 580.2 & 583.6 & 580.4 & 585.5 \\
\hline 45 & 580.7 & 587.7 & 581.6 & 585.7 \\
\hline 46 & 588.0 & 598.0 & 586.5 & 590.9 \\
\hline 47 & 588.7 & 613.8 & 627.0 & 598.1 \\
\hline 48 & 589.0 & 623.5 & 627.3 & 607.0 \\
\hline 49 & 681.5 & 679.9 & 676.6 & 630.4 \\
\hline 50 & 682.1 & 680.4 & 677.5 & 631.9 \\
\hline 51 & 683.1 & 680.7 & 678.1 & 674.2 \\
\hline 52 & 683.4 & 682.0 & 678.6 & 674.7 \\
\hline 53 & 683.6 & 684.1 & 679.3 & 676.0 \\
\hline 54 & 726.8 & 704.6 & 679.9 & 678.3 \\
\hline 55 & 727.2 & 714.5 & 681.4 & 678.6 \\
\hline 56 & 727.8 & 717.3 & 716.6 & 703.6 \\
\hline 57 & 728.1 & 723.9 & 717.0 & 705.9 \\
\hline 58 & 728.2 & 725.1 & 719.3 & 706.4 \\
\hline 59 & 731.7 & 728.1 & 719.8 & 708.0 \\
\hline 60 & 733.2 & 729.9 & 721.7 & 713.6 \\
\hline 61 & 733.4 & 730.4 & 722.2 & 719.1 \\
\hline 62 & 733.8 & 738.1 & 726.0 & 720.8 \\
\hline 63 & 735.0 & 740.4 & 729.0 & 722.4 \\
\hline 64 & 737.5 & 746.4 & 731.6 & 724.6 \\
\hline 65 & 751.0 & 751.1 & 731.7 & 725.1 \\
\hline 66 & 751.0 & 751.5 & 733.0 & 727.6 \\
\hline
\end{tabular}




\begin{tabular}{|c|c|c|c|c|}
\hline 67 & 752.2 & 752.1 & 735.1 & 728.5 \\
\hline 68 & 752.3 & 752.9 & 737.8 & 731.7 \\
\hline 69 & 752.4 & 753.2 & 737.9 & 737.5 \\
\hline 70 & 753.0 & 753.5 & 740.3 & 738.3 \\
\hline 71 & 753.6 & 755.1 & 741.5 & 738.8 \\
\hline 72 & 755.0 & 755.5 & 745.3 & 738.9 \\
\hline 73 & 756.4 & 756.8 & 748.0 & 746.6 \\
\hline 74 & 764.6 & 757.2 & 748.9 & 747.9 \\
\hline 75 & 765.8 & 759.2 & 749.2 & 750.4 \\
\hline 76 & 766.4 & 761.0 & 754.4 & 751.0 \\
\hline 77 & 766.6 & 767.2 & 757.3 & 753.7 \\
\hline 78 & 768.6 & 769.0 & 764.0 & 762.1 \\
\hline 79 & 768.7 & 769.2 & 765.7 & 762.3 \\
\hline 80 & 769.4 & 769.8 & 767.5 & 765.1 \\
\hline 81 & 770.4 & 771.6 & 767.7 & 766.4 \\
\hline 82 & 784.4 & 777.4 & 768.0 & 767.0 \\
\hline 83 & 784.8 & 780.6 & 768.1 & 767.8 \\
\hline 84 & 785.2 & 787.1 & 778.9 & 780.0 \\
\hline 85 & 785.5 & 788.3 & 780.1 & 783.5 \\
\hline 86 & 785.6 & 790.4 & 780.6 & 784.1 \\
\hline 87 & 811.1 & 810.9 & 810.1 & 809.1 \\
\hline 88 & 811.2 & 811.5 & 810.2 & 810.6 \\
\hline 89 & 811.9 & 813.7 & 811.2 & 812.5 \\
\hline 90 & 845.9 & 847.4 & 843.9 & 841.8 \\
\hline 91 & 846.3 & 848.6 & 844.6 & 843.1 \\
\hline 92 & 847.3 & 849.1 & 844.9 & 843.7 \\
\hline 93 & 963.2 & 969.0 & 959.6 & 965.2 \\
\hline 94 & 963.6 & 972.3 & 961.5 & 966.1 \\
\hline 9 & 964.2 & 978.5 & 962.9 & 969.0 \\
\hline
\end{tabular}




\begin{tabular}{|c|c|c|c|c|}
\hline 96 & 968.6 & 978.7 & 966.7 & 970.5 \\
\hline 97 & 969.0 & 980.5 & 968.4 & 972.7 \\
\hline 98 & 969.8 & 980.7 & 969.1 & 978.3 \\
\hline 99 & 970.3 & 981.5 & 973.9 & 988.6 \\
\hline 100 & 977.0 & 982.7 & 978.4 & 996.3 \\
\hline 101 & 1087.0 & 1072.8 & 1084.4 & 1054.6 \\
\hline 102 & 1087.2 & 1090.8 & 1088.7 & 1055.5 \\
\hline 103 & 1087.3 & 1098.4 & 1091.0 & 1095.5 \\
\hline 104 & 1087.7 & 1104.8 & 1093.7 & 1097.0 \\
\hline 105 & 1118.7 & 1123.2 & 1097.1 & 1111.0 \\
\hline 106 & 1119.1 & 1126.6 & 1102.3 & 1113.4 \\
\hline 107 & 1119.4 & 1126.7 & 1115.9 & 1117.8 \\
\hline 108 & 1119.7 & 1128.3 & 1119.1 & 1126.1 \\
\hline 109 & 1120.1 & 1132.9 & 1120.7 & 1126.6 \\
\hline 110 & 1193.2 & 1189.2 & 1185.5 & 1181.9 \\
\hline 111 & 1193.9 & 1200.1 & 1191.0 & 1182.7 \\
\hline 112 & 1194.0 & 1208.1 & 1191.5 & 1187.4 \\
\hline 113 & 1209.7 & 1211.2 & 1206.3 & 1192.1 \\
\hline 114 & 1209.7 & 1221.4 & 1212.2 & 1195.9 \\
\hline 115 & 1210.4 & 1221.7 & 1218.5 & 1204.8 \\
\hline 116 & 1239.2 & 1222.9 & 1218.8 & 1214.2 \\
\hline 117 & 1239.5 & 1234.1 & 1222.9 & 1215.3 \\
\hline 118 & 1239.7 & 1234.9 & 1227.4 & 1218.2 \\
\hline 119 & 1240.0 & 1238.3 & 1233.8 & 1227.4 \\
\hline 120 & 1240.4 & 1248.4 & 1237.0 & 1228.4 \\
\hline 121 & 1268.6 & 1251.3 & 1240.4 & 1236.3 \\
\hline 122 & 1268.7 & 1258.1 & 1245.6 & 1252.0 \\
\hline 123 & 1268.8 & 1262.4 & 1262.6 & 1256.0 \\
\hline 124 & 1269.0 & 1268.8 & 1268.3 & 1265.3 \\
\hline
\end{tabular}




\begin{tabular}{|c|c|c|c|c|}
\hline 125 & 1269.3 & 1278.3 & 1270.1 & 1270.9 \\
\hline 126 & 1299.3 & 1301.7 & 1292.6 & 1284.0 \\
\hline 127 & 1299.5 & 1310.4 & 1304.7 & 1289.1 \\
\hline 128 & 1299.6 & 1310.7 & 1305.7 & 1292.0 \\
\hline 129 & 1325.8 & 1317.4 & 1322.1 & 1313.0 \\
\hline 130 & 1325.9 & 1320.2 & 1325.2 & 1313.7 \\
\hline 131 & 1326.3 & 1322.3 & 1326.3 & 1318.6 \\
\hline 132 & 1326.4 & 1331.0 & 1326.4 & 1320.9 \\
\hline 133 & 1329.0 & 1332.0 & 1328.0 & 1323.7 \\
\hline 134 & 1329.1 & 1334.1 & 1329.0 & 1327.3 \\
\hline 135 & 1329.4 & 1335.7 & 1331.3 & 1331.9 \\
\hline 136 & 1329.5 & 1340.6 & 1333.3 & 1334.7 \\
\hline 137 & 1364.5 & 1343.5 & 1334.7 & 1339.4 \\
\hline 138 & 1364.7 & 1351.8 & 1337.6 & 1342.1 \\
\hline 139 & 1365.1 & 1363.6 & 1351.0 & 1345.1 \\
\hline 140 & 1365.6 & 1369.7 & 1354.7 & 1345.7 \\
\hline 141 & 1366.0 & 1370.7 & 1357.4 & 1350.8 \\
\hline 142 & 1366.0 & 1372.3 & 1357.7 & 1351.1 \\
\hline 143 & 1366.0 & 1374.5 & 1360.6 & 1361.0 \\
\hline 144 & 1366.2 & 1374.6 & 1370.1 & 1369.4 \\
\hline 145 & 1444.9 & 1375.4 & 1372.1 & 1378.7 \\
\hline 146 & 1445.0 & 1379.8 & 1375.7 & 1379.6 \\
\hline 147 & 1445.2 & 1389.0 & 1405.7 & 1382.7 \\
\hline 148 & 1445.3 & 1436.7 & 1406.1 & 1385.3 \\
\hline 149 & 1445.4 & 1438.8 & 1427.5 & 1417.1 \\
\hline 150 & 1450.9 & 1439.1 & 1430.2 & 1418.7 \\
\hline 151 & 1451.5 & 1439.3 & 1440.7 & 1429.9 \\
\hline 152 & 1451.8 & 1440.4 & 1443.2 & 1430.7 \\
\hline 153 & 1453.8 & 1444.5 & 1447.8 & 1435.6 \\
\hline
\end{tabular}




\begin{tabular}{|c|c|c|c|c|}
\hline 154 & 1454.2 & 1455.6 & 1447.8 & 1443.9 \\
\hline 155 & 1454.5 & 1456.7 & 1449.1 & 1454.4 \\
\hline 156 & 1454.6 & 1466.8 & 1451.9 & 1456.7 \\
\hline $157^{b}$ & 1495.9 & 1501.4 & 1488.2 & 1478.6 \\
\hline 158 & 1531.5 & 1508.5 & 1519.3 & 1497.2 \\
\hline 159 & 1531.6 & 1513.5 & 1520.5 & 1500.0 \\
\hline 160 & 1531.8 & 1530.6 & 1520.5 & 1504.1 \\
\hline 161 & 1532.1 & 1538.2 & 1522.5 & 1510.8 \\
\hline 162 & 1561.6 & 1544.6 & 1533.6 & 1514.8 \\
\hline 163 & 1561.9 & 1556.3 & 1550.7 & 1517.7 \\
\hline 164 & 1562.1 & 1560.0 & 1552.5 & 1527.4 \\
\hline 165 & 1600.5 & 1564.0 & 1556.2 & 1538.6 \\
\hline 166 & 1600.5 & 1574.6 & 1559.0 & 1542.1 \\
\hline 167 & 1600.7 & 1579.5 & 1559.2 & 1555.8 \\
\hline 168 & 1601.0 & 1585.4 & 1561.3 & 1557.5 \\
\hline 169 & 1601.3 & 1588.7 & 1574.7 & 1567.6 \\
\hline 170 & 1610.3 & 1593.4 & 1586.4 & 1571.4 \\
\hline 171 & 1610.4 & 1594.3 & 1590.0 & 1575.9 \\
\hline 172 & 1610.5 & 1594.7 & 1591.6 & 1579.2 \\
\hline 173 & 1610.7 & 1597.1 & 1591.8 & 1579.5 \\
\hline 174 & 1611.0 & 1608.1 & 1595.6 & 1588.9 \\
\hline
\end{tabular}




\section{REFERENCES}

(1) Te Velde, G.; Bicke hhaupt, F. M.; Baerends, E. J.; Guerra, C. F.; Van Gisbergen, S. J. A.; Snijders, J. G.; Ziegler, T. Chemistry with ADF. J. Comput. Chem. 2001, 22, 931-967.

(2) Valiev, M.; Bylaska, E. J.; Govind, N.; Kowalski, K.; Straatsma, T. P.; Van Dam, H. J. J.; Wang, D.; Nieplocha, J.; Apra, E.; Windus, T. L., et al. NWChem: A Comprehensive and Scalable Open-Source Solution for Large Scale Molecular Simulations. Comput. Phys. Commun. 2010, 181, 1477-1489. 